\title{
Pattern scaling using ClimGen: monthly-resolution future climate scenarios including changes in the variability of precipitation
}

\author{
Timothy J. Osborn ${ }^{1}$ - Craig J. Wallace ${ }^{1}$. \\ Ian C. Harris ${ }^{1}$ • Thomas M. Melvin ${ }^{1}$
}

Received: 10 March 2014 / Accepted: 11 September 2015 / Published online: 7 October 2015

(C) The Author(s) 2015. This article is published with open access at Springerlink.com

\begin{abstract}
Development, testing and example applications of the pattern-scaling approach for generating future climate change projections are reported here, with a focus on a particular software application called "ClimGen". A number of innovations have been implemented, including using exponential and logistic functions of global-mean temperature to represent changes in local precipitation and cloud cover, and interpolation from climate model grids to a finer grid while taking into account land-sea contrasts in the climate change patterns. Of particular significance is a new approach for incorporating changes in the inter-annual variability of monthly precipitation simulated by climate models. This is achieved by diagnosing simulated changes in the shape of the gamma distribution of monthly precipitation totals, applying the pattern-scaling approach to estimate changes in the shape parameter under a future scenario, and then perturbing sequences of observed precipitation anomalies so that their distribution changes according to the projected change in the shape parameter. The approach cannot represent changes to the structure of climate timeseries (e.g. changed autocorrelation or teleconnection patterns) were they to occur, but is shown here to be more successful at representing changes in low precipitation extremes than previous pattern-scaling methods.
\end{abstract}

This article is part of a Special Issue on "The QUEST-GSI Project" edited by Nigel Arnell

Electronic supplementary material The online version of this article (doi:10.1007/s10584-015-1509-9) contains supplementary material, which is available to authorized users.

Timothy J. Osborn

t.osborn@uea.ac.uk

1 Climatic Research Unit, School of Environmental Sciences, University of East Anglia, Norwich NR4 7TJ, UK 


\section{Introduction}

For most scenarios of future climate change (e.g. Christensen et al. 2013), the detailed patterns (geographical, seasonal and multi-variable structure) are commonly derived from simulations with general circulation models (GCMs) or regional climate models (RCMs). The magnitude of the change is not always obtained from the same GCM or RCM simulation, however, because the patterns may be scaled to represent (i) cases with different sensitivities of climate to greenhouse gas forcing (Heinke et al. 2013); (ii) cases with different future emissions or concentrations of greenhouses gases, and hence different future global temperature changes (Huntingford et al. 2010); or (iii) time periods for which suitable simulations have not been undertaken (e.g., to use RCM simulations of the late 21st century to provide projections for the early or mid 21st century, Cabré et al. 2010, or to use equilibrium GCM simulations to provide transient projections, Murphy et al. 2009). This combination of deriving a pattern (usually expressed in a normalised way, such as change per degree Celsius of global-mean temperature change) and then scaling its magnitude is commonly called "pattern scaling" (PS). The globalmean temperature change (i.e. the scaling factor) is typically set to a specific warming level or simulated by a simple climate model designed either to explore uncertainty in global temperature projections or to emulate GCM global warming. PS was initially introduced to enable the creation of transient climate projections from the equilibrium response of a GCM to a doubling of $\mathrm{CO}_{2}$ concentration (Santer et al. 1990), but it remains an important technique for generating projections, especially for probabilistic approaches to dealing with uncertainty (e.g. New and Hulme 2000; Watterson and Whetton 2011).

Despite the widespread use of PS, the underlying assumption of a linear relationship between local climate change and global-mean temperature change that is invariant under a range of rates and amounts of warming has received relatively little critical attention until recently. The most comprehensive early assessments (Mitchell et al. 1999; Mitchell 2003) found that statistically significant non-linearities could be identified with careful use of ensembles of simulations, but that the errors that would thus result from using PS are small compared with the many other uncertainties that exist in future climate scenarios. More recent assessments, facilitated by ensembles of individual and multiple models, confirm that PS can approximately emulate the GCM-simulated response to increasing greenhouse gas concentrations (e.g. Tebaldi and Arblaster 2014; Heinke et al. 2013) but that it performs less well for scenarios when the forcing stabilises (Tebaldi and Arblaster 2014) or there are strong differences in regional forcings, such as anthropogenic aerosols (Ishizaki et al. 2014 show significant precipitation differences downwind of aerosol-affected oceans).

The primary aim of this paper is to present some new PS developments (section 2), particularly for incorporating changes in precipitation variability which are ignored in climate change impact assessments that are based on simple climate projection approaches such as PS or the "delta-change" method. The new developments have been implemented in the "ClimGen" software to generate the pattern-scaled climate projections that have been used in multiple projects (QUEST GSI project, Arnell et al. 2014; Tyndall Centre integrated assessment system, Warren et al. 2008; and AVOID project, Arnell et al. 2013; Warren et al. 2013). A secondary aim, therefore, is to describe the specific approaches as they have been implemented in ClimGen (section 2 and the Supplementary Information, SI), and to illustrate some of the possible applications of ClimGen (section 4) for studying the influence of precipitation variability and for exploring the results of multi-model ensembles. An assessment of the reliability of PS is not the main purpose of this paper (for this, see the studies referenced 
earlier) but some simple measures of pattern stability between scenarios/simulations that are informative on this point are presented in section 3.

\section{Climate projections using pattern scaling}

\subsection{Pattern scaling assumptions and calculations}

The basic assumption underlying the PS approach is that there is a linear (perhaps after a simple transformation) relationship between local climate change and global-mean temperature change $(\Delta T)$ that is invariant under the range of climate changes reasonably expected during the coming century or more. This unknown pattern might be estimated from a GCM simulation, with a different pattern obtained from each GCM considered. Biases in a GCM's simulation of present-day climate are typically ignored by using only the climate change pattern, and applying this to an observed present-day climatology (thus similar to the "deltachange" method).

This is illustrated in SI Figure S1, comparing different time periods and scenarios. SI section S2 describes how the "normalised" patterns (local change per ${ }^{\circ} \mathrm{C}$ of global warming) are diagnosed for use in ClimGen (see also SI section S6 for a mathematical description), using a regression approach of running-mean data pooled across initialcondition ensembles and multiple scenarios. A modified interpolation from different GCM grids to a common grid that provides finer detail near to coasts in the presence of a strong land-sea contrast in climate change is also illustrated (SI Figure S2).

\subsection{Combining mean climate changes with observed variability}

For any given global-mean temperature rise, the climate change for a given calendar month and climate variable can be estimated by:

$$
\Delta \boldsymbol{V}=\boldsymbol{a} \Delta T
$$

where $\boldsymbol{a}$ is the normalised pattern for that month and variable from a selected GCM, and $\Delta \boldsymbol{V}$ is the field of climate change obtained. For some applications this is sufficient, but many users require a plausible sequence of "weather" (at the monthly timescale, at least) comprising the climate change added to the initial climate field $\left(\overline{\boldsymbol{V}_{\mathbf{0}}}\right)$ and a sequence of anomalies from the mean climate. In ClimGen, the latter is obtained by using a sequence from a monthlyresolution observed climate dataset. Using the observed record has the advantage that it contains realistic spatio-temporal structures on large scales (e.g. those associated with major modes of climate variability), which may be poorly represented using alternatives such as weather generators or direct (even bias-corrected) GCM output. Its disadvantage is that this unforced monthly climate variability does not change even though the underlying mean climate is changing. For precipitation this limitation is partly overcome in section 2.3 , and a similar approach could be taken for other variables.

A future sequence of monthly "weather" under a changed climate can be generated according to:

$$
\boldsymbol{V}_{\boldsymbol{t}}=\overline{\boldsymbol{V}_{0}}+\boldsymbol{V}_{\boldsymbol{t}}^{\prime}+\boldsymbol{a} \Delta T_{t}
$$

where $\boldsymbol{V}_{\boldsymbol{t}}^{\prime}$ is the field of observed anomalies in year $t$ for the given month. $\Delta T_{t}$ is now a global-mean temperature change specific to year $t$; thus it can represent a transient timeseries 
of warming, or if all values are equal it can be used to generate a sequence of unforced monthly climate variability representative of climate under a specific level of global warming (e.g. $\left.\Delta T_{t}=2^{\circ} \mathrm{C}\right)$.

For precipitation, the climate change can also be applied in a multiplicative rather than additive way (see e.g. Osborn 1997 for some advantages), so that the future sequence of mean precipitation fields is given by this modification to Eq. 2:

$$
\boldsymbol{V}_{\boldsymbol{t}}=\overline{\boldsymbol{V}_{0}} \cdot \boldsymbol{V}_{\boldsymbol{t}}^{\prime} \cdot\left(1+\boldsymbol{a} \Delta T_{t}\right)
$$

where the observed anomalies are now expressed as fractional deviations (i.e. $\overline{\boldsymbol{V}_{\mathbf{0}}}$ is divided into each observed value rather than subtracted from it) and $\boldsymbol{a}$ is the normalised pattern of relative change in precipitation from the 1961-1990 mean simulated by that GCM for the given month. The absolute lower limit of zero precipitation is violated in regions of decreasing precipitation $(a<0)$ if $\Delta T>-1 / a$ (which could occur if projections are extrapolated to larger temperature changes). In ClimGen, any negative precipitation predictions are simply replaced with zero, but a further option is to use an exponential function of global temperature to avoid this problem:

$$
\boldsymbol{V}_{\boldsymbol{t}}=\overline{\boldsymbol{V}_{0}} \cdot \boldsymbol{V}_{\boldsymbol{t}}^{\prime} \cdot \exp \left(\boldsymbol{a} \Delta T_{t}\right)
$$

The normalised pattern of change, $\boldsymbol{a}$, is obtained by regressing logarithms of the simulated future mean precipitation (relative to the simulated 1961-1990 mean) against the global-mean temperature (see SI section S6). This approach should be used cautiously because where $a>0$ precipitation increases accelerate with increasing $\Delta T$. ClimGen also allows a mixed form, therefore, using an exponential function of global temperature for drying regions/months and a linear function for wetting. This is clearly ad hoc (because there is no reason why the functional form should change just at the present-day climate value), but an analysis of six earlier-generation GCMs (not shown) suggested that a close match to the GCM-simulated changes in precipitation could be obtained and others (Watterson and Whetton 2011) have also used a mixed form.

For cloud cover, there are both upper (100\%) and lower (0\%) physical limits. As with precipitation, ClimGen simply truncates any future values that lie outside this range. A second option is also provided, however, which is to use logistic regression between cloud cover and $\Delta T$ to derive the normalised patterns of cloud cover change, and to combine the scaled cloud cover change with the observed mean climate and anomalies in logit space prior to conversion via the logistic function to yields values between 0 and 1 only (or 0 and $100 \%$ when scaled by $100)$ :

$$
V_{t}=100 /\left(1+\exp \left[-\ln \frac{\overline{V_{0}}+V_{t}^{\prime}}{1-\overline{V_{0}}+V_{t}^{\prime}}-a \Delta T_{t}\right]\right)
$$

In practise, it was found that this additive-change logistic approach only differed from the linear approach (Eq. 2) in a few regions where the observed cloud cover was close to 0 or $100 \%$. Heinke et al. (2013) use a similar approach for pattern-scaling changes in the frequency of wet months. 
In ClimGen, the fields of the initial climate (1961-1990 mean) and of the observed anomalies can be selected from different versions of the CRU TS land-surface dataset (Harris et al. 2014), on a $0.5^{\circ}$ longitude by $0.5^{\circ}$ latitude grid. For sea surface temperature (SST, the only variable included over the oceans) the HadISST1 (Rayner et al. 2003) data are provided after interpolation to the same grid. In all cases, the monthly anomalies were detrended by regressing the decadally-smoothed observed timeseries against the decadally-smoothed observed global-mean temperature prior to being used in ClimGen. The rationale for this is that the climate change signal is being provided by the pattern-scaling component and the superimposed variability $\left(\boldsymbol{V}_{\boldsymbol{t}}^{\prime}\right)$ should not add an additional trend.

\subsection{Changes in precipitation variability}

Climate model simulations indicate that climate variability (especially precipitation) may change with climate state, but the methods described above (where sequences of observed anomalies are used to represent future climate variability) do not allow any changes in future climate variability. The absolute-change method (Eq. 2) leaves the standard deviation of the observed anomalies unchanged for the future projection, whereas the relative-change methods (Eqs. 3 or 4 ) alter the standard deviation in proportion to the change in the mean (i.e. the coefficient of variation is constant). A modification of these methods has been developed to overcome this limitation for precipitation, which could be adapted for other variables as well. Figure 1 illustrates some components of this approach.

It is convenient to separate the high-frequency and low-frequency variations, with modifications to the former to represent changes in climate variability, while modifications to the latter are included in the change in the mean climate. An observed timeseries $(X)$ of monthly precipitation (e.g. from the CRU TS3.10 dataset, Harris et al. 2014), for a single grid cell and month of the year, is smoothed with a Gaussian-weighted filter to obtain the low-frequency series $\left(X_{L}\right)$. The high-frequency component is expressed as fractional deviations from this lowfrequency component:

$$
X_{H}=X / X_{L}
$$

which has the advantage that it is stationary, has only positive values and a mean close to 1 , and in most locations can be approximately fitted by a gamma distribution with shape and scale parameters $[\alpha, 1 / \alpha]$ (the mean of a gamma distribution is the product of the shape and scale parameters). Each value in the observed high-frequency fractional deviation timeseries can then be converted to a cumulative probability $\left(X_{P}\right)$ using the cumulative density function (CDF) of the $[\alpha, 1 / \alpha]$ gamma distribution, such that $X_{P}$ is the probability of obtaining a value less than or equal to $X_{H}$.

The GCM-simulated monthly precipitation timeseries were analysed in a similar way (i.e., a gamma distribution was fitted to high-frequency fractional deviations) within a sliding 49-year window, pooling data from across multiple ensemble members where available, to obtain a timeseries of $\alpha$ for that grid cell and month of the year. These were regressed against 49-year running means of simulated $\Delta T$, to obtain normalised patterns of change in precipitation shape, $\alpha$. That is, PS is applied to this measure of the change in the shape of the precipitation distribution, in the same way that it is applied to the change in the mean precipitation. Since $\alpha$ is bounded at zero, the relative-change exponential function (Eq. 4) is used in regions/months where $\alpha$ decreases during the GCM simulation 
(a) shp J multi-model mean

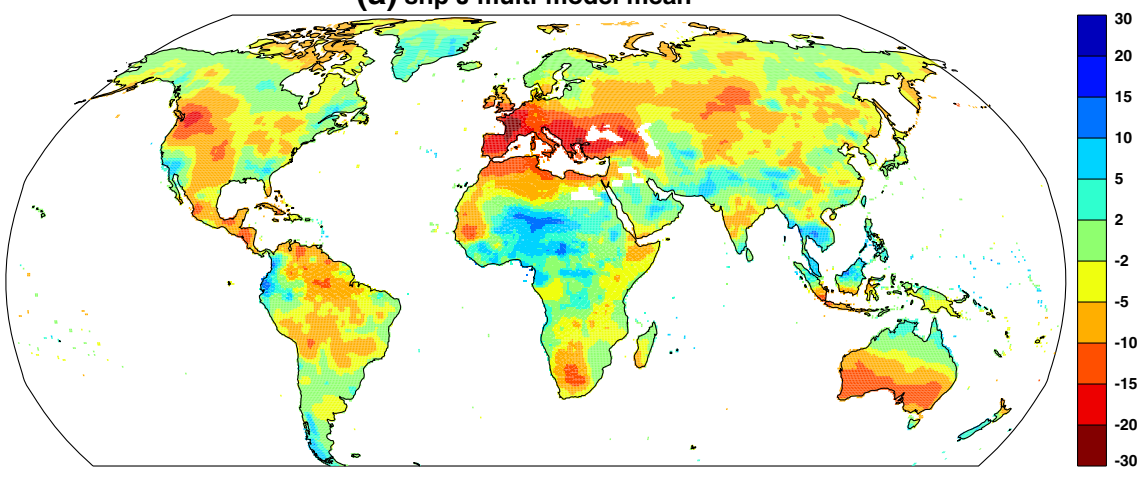

(b)

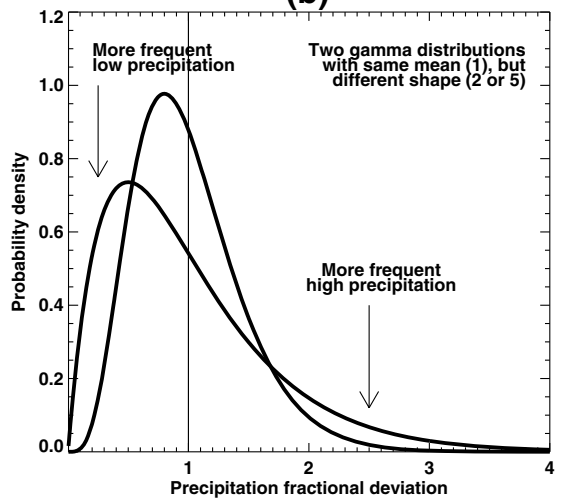

(d) SEE July (dt=0 \& 3, fixed gamma)

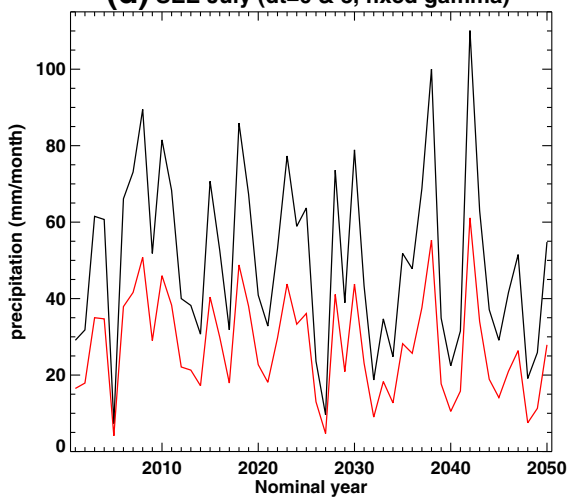

(c) SEE JuI

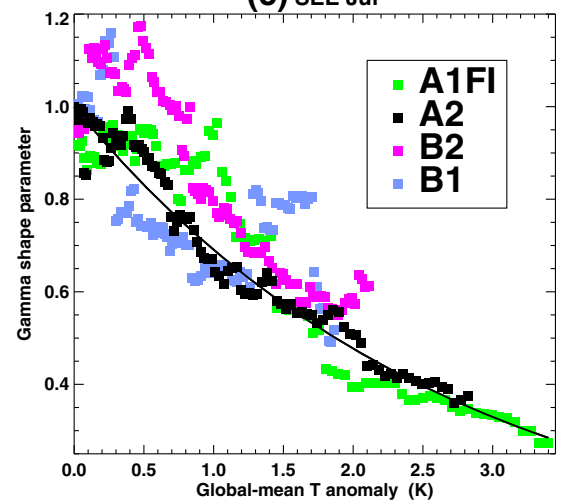

(e) SEE July ( $d t=3$, fixed \& changed gamma)

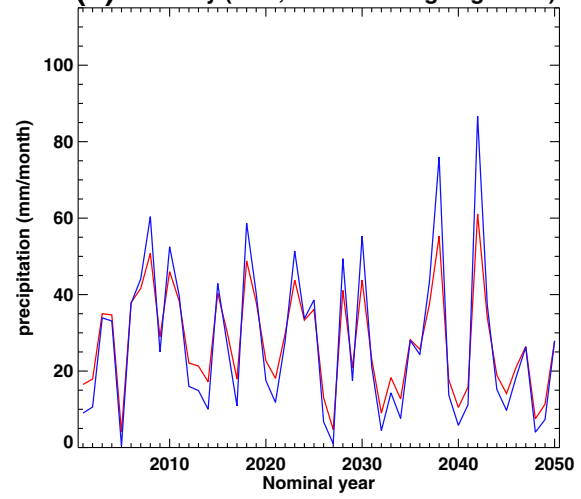

(e.g. Fig. 1c), and the relative-change linear function (Eq. 3) is used where $\alpha$ increases (see earlier discussion). 
Fig. 1 Illustrating the perturbation of monthly precipitation variability via changes to the shape $(\alpha)$ of the gamma distribution. a Multi-model mean of 22 CMIP3 GCMs normalised pattern of change in $\alpha$ for July precipitation $\left(\%\right.$ change $\left./{ }^{\circ} \mathrm{C}\right)$. b Gamma distributions with the same mean but different $\alpha$ values: $\alpha=2$ gives more frequent lower and higher values than $\alpha=5$. $\mathbf{e}$ Dependence of southeast England (SEE) July precipitation $\alpha$ on global-mean temperature change calculated from 49-year sliding windows of July precipitation simulated by the HadCM3 GCM under four different SRES scenarios. Each value of $\alpha$ has been divided by the 1950-1999 reference period $\alpha$, so values $<1$ indicate reductions in $\alpha$. The black line shows an exponential function fit to the SRES A2 scenario data. d SEE July precipitation from the CRU TS3.00 data (black) and scaled by 0.55 (red) to represent a $\Delta T=3$ scenario using the HadCM3 pattern. $\mathbf{e}$ The future precipitation scenario in (d) is repeated (red) and compared with a series with the same mean but a $62 \%$ reduction in $\alpha$ (blue)

To construct a precipitation scenario for a given $\Delta T$, PS is used to obtain both the relative change in mean precipitation and the relative change in gamma shape parameter $(\Delta s)$. The latter is used to perturb each observed precipitation anomaly, by finding the value that has the same cumulative probability as $X_{P}$ but in the perturbed gamma distribution with shape and scale parameters $[\Delta s \cdot \alpha, 1 /(\Delta s \cdot \alpha)]$. Note that this perturbed distribution still has a mean of 1 , since we are not dealing with changes in mean precipitation in this component. We find the high-frequency fractional deviation of precipitation for a particular value in the timeseries that is representative of the future climate $\left(X_{F}\right)$ such that:

$$
\mathrm{P}\left(Y \leq X_{H}\right)=X_{P}=\mathrm{P}\left(Z \leq X_{F}\right)
$$

where $Y \sim \operatorname{Gamma}(\alpha, 1 / \alpha)$ and $Z \sim \operatorname{Gamma}(\Delta s \cdot \alpha, 1 /[\Delta s \cdot \alpha])$. The pattern-scaled projection, with perturbed precipitation variability, can be assembled by replacing $\overline{\boldsymbol{V}_{\mathbf{0}}}$ in Eqs. 3 or 4 by the field of $X_{L}$ for year $t$ (since the low-frequency component includes the mean, absolute precipitation) and $\boldsymbol{V}_{\boldsymbol{t}}^{\prime}$ by the field of $X_{F}$ for year $t$.

CMIP3 GCMs (Table S1) simulate changes in precipitation gamma shape parameter that are consistent in sign in some regions, but not in others. Most CMIP3 GCMs simulate a reduction in $\alpha$ in regions with a Mediterranean-type climate and this is apparent in the multimodel mean change (Fig. 1a), which could be important for the occurrence of both dry and wet extremes (Fig. 1b). Taking an example from a grid cell over southeast England and one specific GCM (HadCM3), we find a strong reduction in $\alpha$ for July precipitation as $\Delta T$ increases in different scenarios (Fig. 1c) that can be approximately represented by an exponential function.

Using this function and an equivalent one for mean July precipitation simulated by the same GCM, Fig. 1d and e illustrate the application of the technique for $\Delta T=3{ }^{\circ} \mathrm{C}$. The observed monthly precipitation timeseries has been modified according to the HadCM3 model response (Fig. 1d changing only the mean; 1e also changing the distribution shape) to produce a timeseries appropriate for a climate under $\Delta T=3{ }^{\circ} \mathrm{C}$; the $x$-axis years are nominal. For this amount of global warming, the HadCM3 GCM pattern gives a $45 \%$ reduction in mean July precipitation, which is applied to the observed precipitation (black line) to yield a sequence representative of the future climate (red line; as noted earlier, the relative-change method lowers the standard deviation of the monthly anomalies in proportion to the change in the mean). However, the HadCM3 pattern also gives a $62 \%$ reduction in the gamma shape parameter: when this is applied to the precipitation series the mean is not altered further but the distribution becomes more skewed and its standard deviation increases (blue line), as expected from Fig. $1 b$. 


\section{Assessing pattern scaling performance}

Given the complex nature of the interactions represented in GCMs, it is not surprising that, given enough data (e.g. initial-condition ensembles to separate signal from noise), it is possible to falsify the assumption of an invariant, linear relationship between local and global climate change that is the basis for PS (Mitchell 2003). It should be viewed as an approximation rather than an assumption, therefore, and the more interesting question is how accurate is this approximation in practical terms. A useful measure of PS performance is the size of the error in this linearity approximation relative to the other sources of uncertainty in future climate projections. Since PS is typically employed to explore the spread of possible future climates represented by a multiGCM ensemble, we present an estimate of the pattern-scaling error relative to this spread.

Although the "standard" pattern used in ClimGen is based on pooling data from multiple scenario simulations (see SI), we also diagnosed normalised patterns from separate CMIP5 simulations for the four RCPs (Representative Concentration Pathways: van Vuuren et al. 2011). The pattern-scaling approximation is that, for a particular GCM, these patterns should be similar regardless of scenario (of course they will not be identical even if the approximation is valid, because they are each affected by the internal variability that differs in each simulation). For the 21 CMIP5 GCMs analysed here, we have 71 alternate patterns (not 84 , since not all GCMs have simulated all four RCP scenarios). For each variable, land grid cell and month of the year, we therefore have 71 normalised changes (local change per degree of global warming) whose total variance represents the combined "between-GCM" variance and the "within-GCM" variance (i.e. the differences between the four RCP-specific patterns for each GCM). Figure 2 shows the percentage of the total variance attributed to "within-GCM" variance for changes in annual-mean temperature and precipitation. Tebaldi and Arblaster (2014) use a similar approach to partition the standard deviation between models and scenarios.

For annual temperature there are few regions where the differences between the four RCP-specific patterns contributes more than $20 \%$ of the overall variance, even around the North Atlantic or the Arctic where we might expect a stronger influence of nonlinear changes in the thermohaline circulation, sea ice or snow (Ishizaki et al. 2012). For some individual months (not shown), there are more extensive regions where this measure of poor pattern-scaling performance is between 20 and $40 \%$ of the variance. However this may simply reflect the greater uncertainty in each pattern arising from internal variability for months compared with annual means. The latter source of error may also contribute to the greater within-GCM variance apparent for annual precipitation (Fig. 2b), with extensive areas of the subtropics and mid latitudes having more than $20 \%$ of the total variance associated with differences between RCP-specific patterns. It is likely that these higher contributions also indicate poorer performance of the invariant linear approximation (and therefore of PS) for precipitation than for temperature, though almost everywhere the remaining variance (arising from differences between CMIP5 GCMs) is the biggest component. Finally, we note that this measure of PS performance is considerably better (i.e. lower values) for the relative changes in precipitation shown in Fig. $2 \mathrm{~b}$ than for absolute changes (not shown). This is one reason to prefer the relative approach (Eq. 3) over the additive approach (Eq. 2) for precipitation PS. 

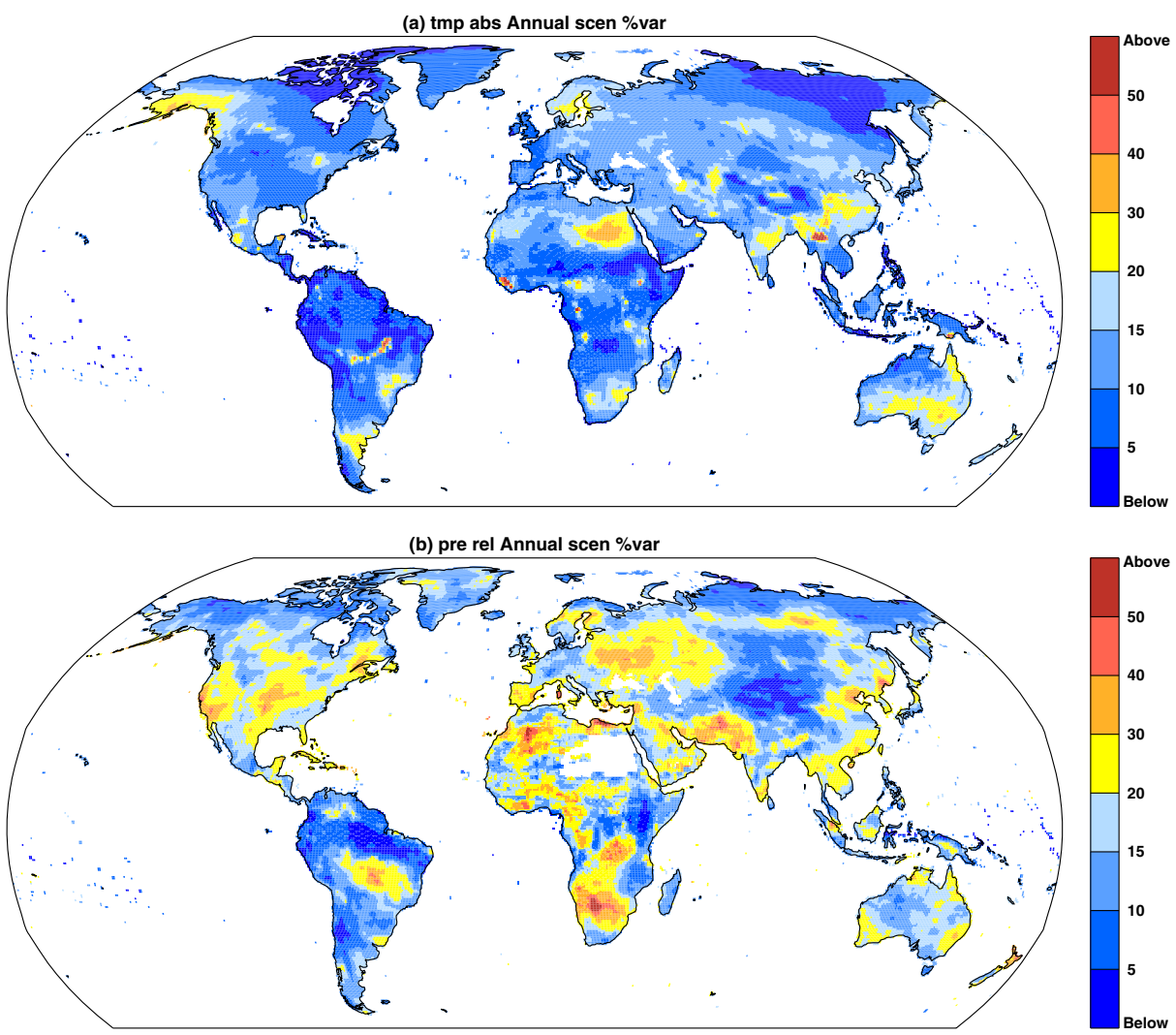

Fig. 2 Inter-scenario differences relative to inter-model differences for the CMIP5 multi-model ensemble. The percentage of the total variance in local normalised changes in annual-mean (a) temperature and (b) precipitation arising from within-GCM differences (i.e. differences between patterns diagnosed from individual RCP scenario simulations for each GCM). The total variance is calculated over the 71 different normalised patterns, and then recalculated after first subtracting each model's mean (across the individual RCP patterns for that GCM) to obtain the within-GCM component. The remaining component (i.e. $100 \%$ minus the value shown) is associated with between-GCM differences

\section{Applying pattern scaling using ClimGen}

\subsection{Introduction}

The approaches developed and reported in this paper for generating climate projections by combining a pattern-scaled climate change signal with unforced variability from observations (perturbed to represent future changes in variability) support a wide range of potential applications concerned with exploring the spread of impacts arising from changing climate. Patterns have been diagnosed from the CMIP3 (Table S1; Meehl et al. 2007), CMIP5 (Table S2; Taylor et al. 2012) and QUMP (Murphy et al. 2004) ensembles. SI section S3 describes two ensemble approaches that allow the climate change influence to be isolated from the unforced variability in these ClimGen projections, so that climate change impacts can be more precisely quantified. SI section S5 uses ClimGen to compare the multi-model mean patterns of climate change simulated by the CMIP3 and CMIP5 ensembles, whereas in the next two sections we demonstrate the importance of changes in variability for the projection of 
precipitation extremes and explore the spread in results from ensembles of model patterns, respectively.

\subsection{Projections of dry-month frequency with and without changes in precipitation variability}

Nearly all the CMIP3 and CMIP5 GCMs simulate reductions in summer precipitation gamma shape (i.e. increased variability and skew, Fig. 1) in southern Europe, so this region is used to illustrate the importance of these changes and the ability of the new methods presented here to more closely emulate the underlying GCM projections. Figure 3 shows the projected future frequency of very dry August months (defined as those below the 1951-2000 6th percentile) for ClimGen projections with and without changes in precipitation variability and for the direct GCM simulations, using the CMIP5 GCM MPI-ESM-MR under the RCP8.5 scenario. ClimGen patterns diagnosed from this GCM were scaled by the global temperature changes simulated by MPI-ESM-MR for RCP8.5. All three show increased frequency (fraction of dry months $>0.06$ ) for much of southern to mid-latitude Europe, but these are more pronounced and reach further north when precipitation variability changes are included in ClimGen (Fig. 3b) or implicitly in the GCM projection (Fig. 3c). Increases in mean August precipitation in the high latitudes are linked to reduced occurrence of very dry months, but this is reversed in some regions (e.g. NE Europe) by changes in variability that increase the dry tail of the distribution despite the increased mean.

Differences can be seen more easily when the changing frequency of dry months is averaged over southern Europe (Fig. 3d). The ClimGen analysis shows that only about half of the increased dry-month frequency in this region arises from reduced mean precipitation (red curve) - the remaining increase (black curve) is only obtained when changes in the variability (i.e. distribution shape) are included in making the PS projections. The results for 50-year running windows (thick lines) also demonstrate that the new PS method yields a close match to the direct GCM results (blue curve). There are differences between the GCM and PS results (e.g. the GCM values are lower around 2040 and higher at the end of the analysis in 2075). With only a single MPI-ESM-MR RCP8.5 simulation, it is not possible to determine if they arise from nonlinear behaviour that PS cannot capture or simply from unforced variability in the GCM run, but it is clear that the inclusion of precipitation variability changes is essential to get close to the GCM results. These results are explored further in SI section S4 for other percentile definitions of dry months.

Fig. 3 Projected changes in dry August months with and without changes in precipitation variability compared with direct GCM results, for the RCP8.5 scenario and the MPI-ESM-MR GCM. Fraction of August months during 2051-2100 that are "dry" in projections from (a) ClimGen changing only mean precipitation; (b) ClimGen changing both precipitation mean and variability; and (c) direct GCM output. Dry months are where precipitation is less than the 6th percentile of the 1951-2000 ClimGen or GCM data. Values below (above) 0.06 represent less (more) frequent dry August months in 2051-2100 than 1951-2000 (areas with too many zero precipitation months during the reference period to define the 6 th percentile are left white). (d) Timeseries of the fraction of dry months averaged over the region defined by the black rectangle in the other panels for 10-year (thin lines) and 50-year (thick lines) running windows, plotted against the central year of each window. Blue: GCM; red: ClimGen mean changes only; black: ClimGen mean and variability changes. Only one simulation is available for the GCM, whereas for ClimGen the 10-year running window results are based on an ensemble of 5 projections for 2001-2100 (each appended to the 1951-2000 CRU TS observations), showing the ensemble mean (thin lines) and \pm 2 standard deviation (SD) range (grey shading, only for changes in both mean and variability) 
(a) gamfix Aug 6\%ile 2051-2100
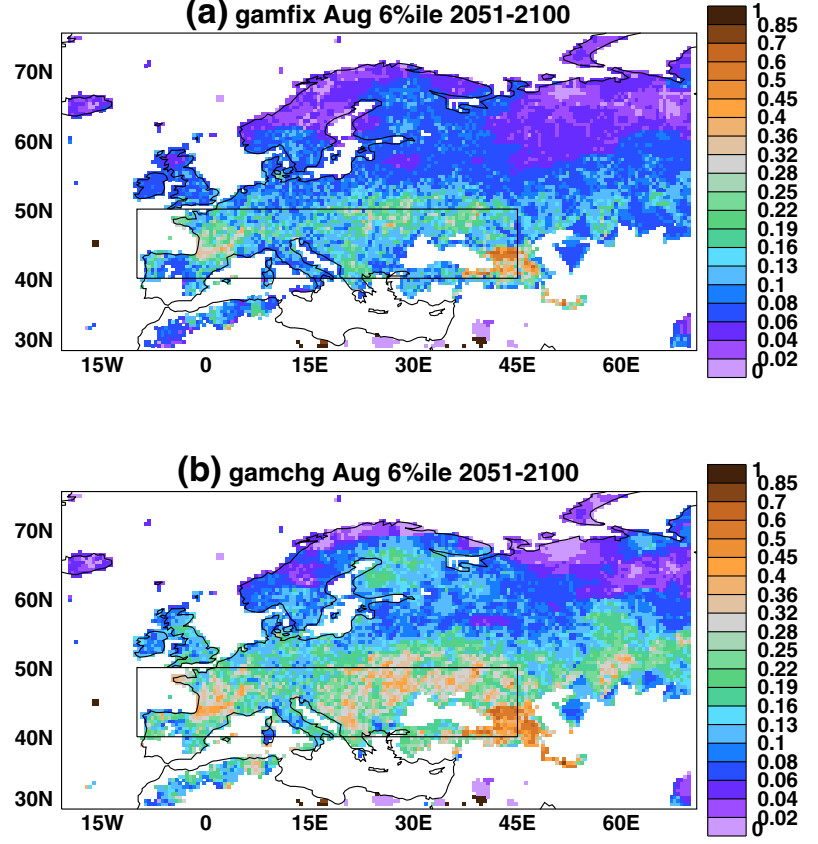

(c) GCM Aug 6\%ile 2051-2100

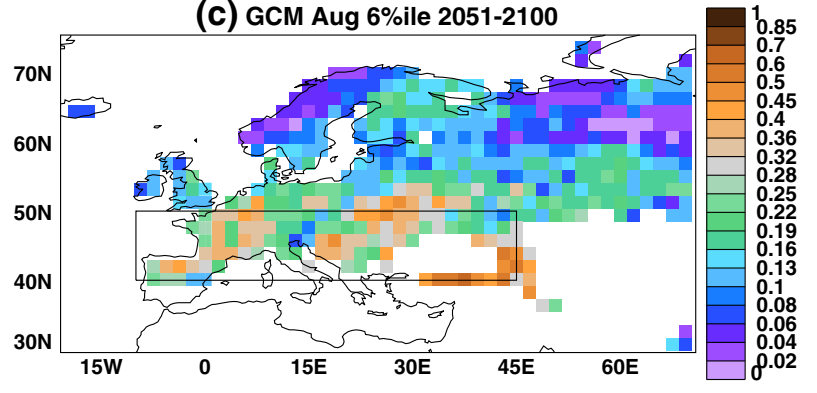

(d) Aug 6\%ile

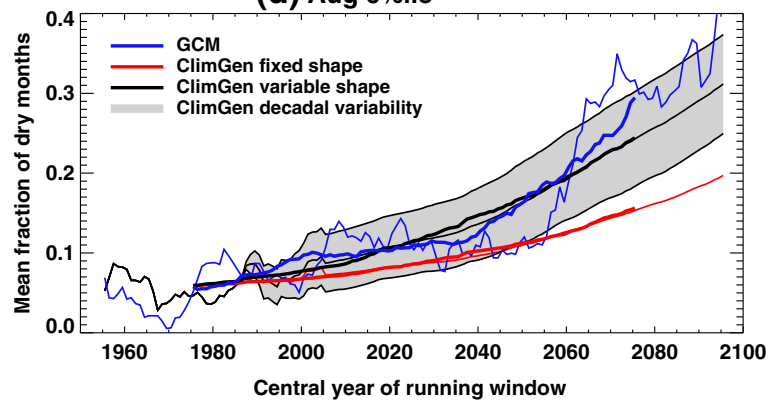


Unforced variability is much greater when a shorter, 10-year running window is used, so much so that the single-run GCM results cannot be relied upon to represent climate changes with such fine time resolution (thin blue line in Fig. 3d). For example, we should not conclude that climate change will hardly alter the occurrence of very dry August months in southern Europe out to the 2050s but they will then rapidly double in frequency one decade later. Using the techniques developed in SI section S3 and implemented in ClimGen, we can generate an ensemble of PS projections using the same patterns of mean and variability changes but with the underlying observed anomalies shifted in time by different amounts. We can then analyse the ensemble of projections in a sliding 10-year window (thin red and black lines) and obtain very similar results as for the 50-year window but with the ability to extend the analysis up to the final decade of the projections rather than just the final 50 years. The spread between ensemble members (grey shading for the case with changes in both mean and variability) is also useful to estimate the decadal variability, though in this case study it does not appear to be as strong as the GCM-simulated decadal variability, possibly because the 50 years of observed precipitation used to provide the variability for the PS projections is not sufficient to fully sample decadal variability. Note also that this spread is not an uncertainty estimate for the climate change signal - the latter is best explored by comparing results from multiple GCMs, a task that ClimGen facilitates.

\subsection{Exploring model spread in two model ensembles}

Our final example is the comparison of two model ensembles for exploring part of the uncertainty in future projections, with PS applied for a range of fixed global-temperature increases rather than a transient scenario. The future climate fields are then averaged spatially to represent national averages and temporally to represent 30 -year mean seasonal climates. Figure $4 \mathrm{a}, \mathrm{b}$ shows changes relative to the present-day for India for two seasons (DecemberFebruary, DJF, and June-August, JJA), based on just one CMIP3 GCM (NCAR CCSM 3.0). The linear dependence of national-average temperature change on global-mean temperature change is a direct result of the linear PS approximation. The actual GCM results will differ from these because the linearity assumption may be a poor approximation for this GCM and the GCM results exhibit internal climate variability (therefore any differences cannot be entirely attributed to a failure of the PS approximation). The precipitation changes show small deviations from linearity, arising from the exponential relationship with global-mean temperature that is assumed where the GCM simulates a reduction in mean precipitation (Section $2 b$ ).

The patterns for this GCM show winter warming that is above the global-mean change by a factor of around 1.4, while summer warming is about 0.8 of the global-mean change. This GCM simulates decreased winter precipitation (though small in absolute terms, given that present-day Indian winter precipitation is already low) but a moderate increase in rainfall

Fig. 4 Changes in seasonal (left: DJF; right: JJA) temperature ( $x$-axis, ${ }^{\circ} \mathrm{C}$ change from $1961-1990$ mean) and precipitation ( $y$-axis, as a ratio of the 1961-1990 mean) estimated by pattern scaling and then averaging over all grid cells in India. Black dots show the variability in 30-year means of these quantities simulated in a 1000-year control simulation with the HadCM3 GCM. (a,b) For one CMIP3 GCM (NCAR CCSM3.0) and six different global-mean temperature changes $\left(0.5\right.$ to $5^{\circ} \mathrm{C}$, as labelled). (c,d) For 22 CMIP3 GCMs (see key in (c)) for global-mean temperature changes of $1{ }^{\circ} \mathrm{C}$ (left-hand cluster of symbols) and $4{ }^{\circ} \mathrm{C}$; (e,f) Comparing the interGCM spread from the CMIP3 (coloured symbols and pink shading) and QUMP (black letters and blue shading) ensemble for global-mean temperature increase of $4{ }^{\circ} \mathrm{C}$. The shading indicates the $\pm 2 \mathrm{SD}$ ranges (and coloured lines mark the \pm 1 SD ranges) under the assumption of a bivariate normal distribution (or a log-normal distribution if a logarithmic transformation reduces the skewness of the data) 
(a) India DJF

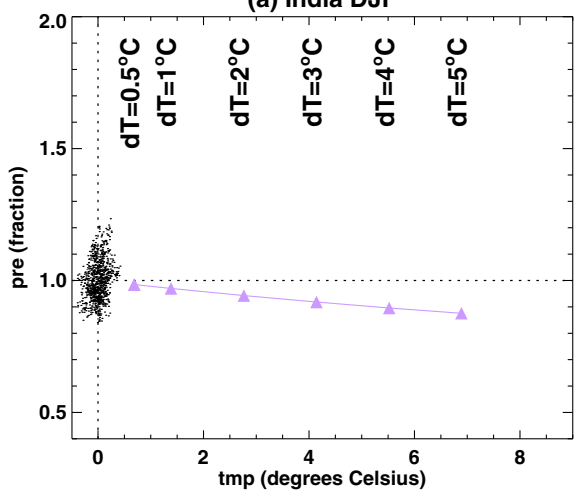

(c) India DJF

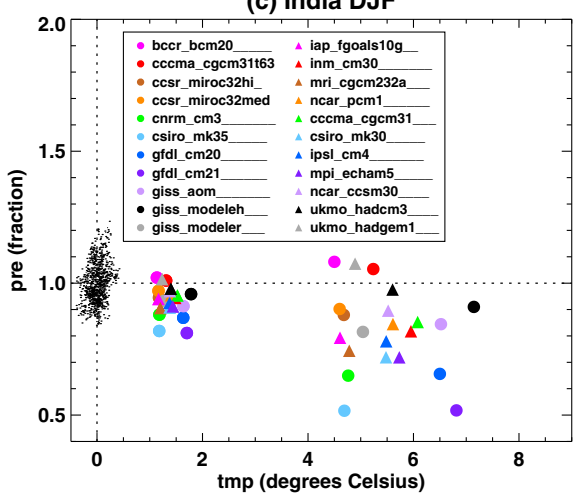

(e) India DJF

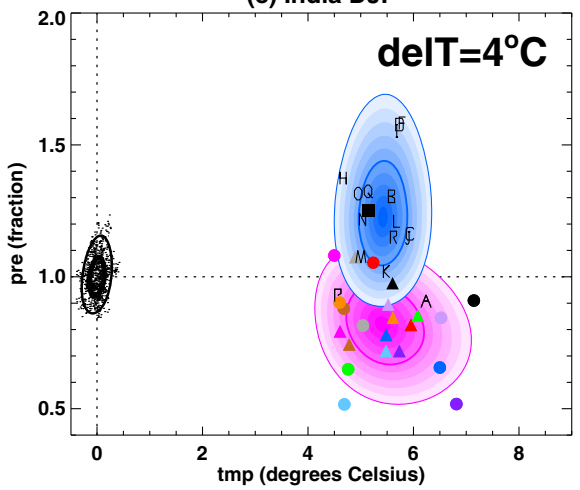

(b) India JJA

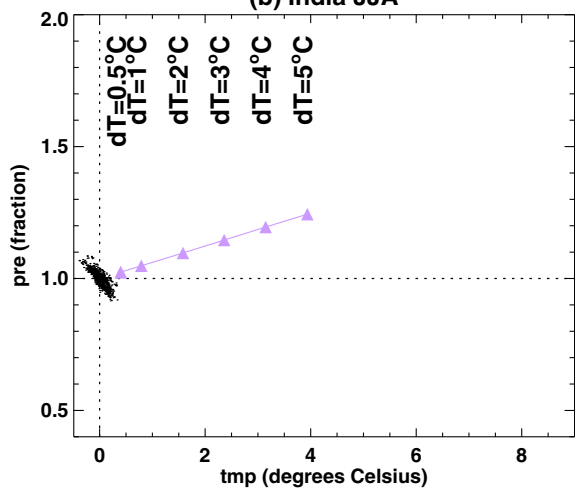

(d) India JJA

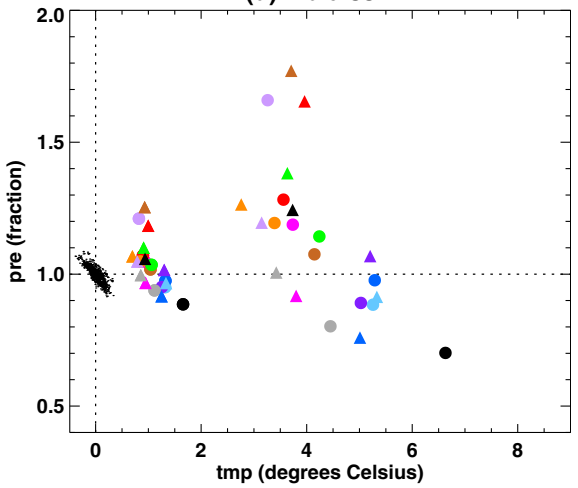

(f) India JJA

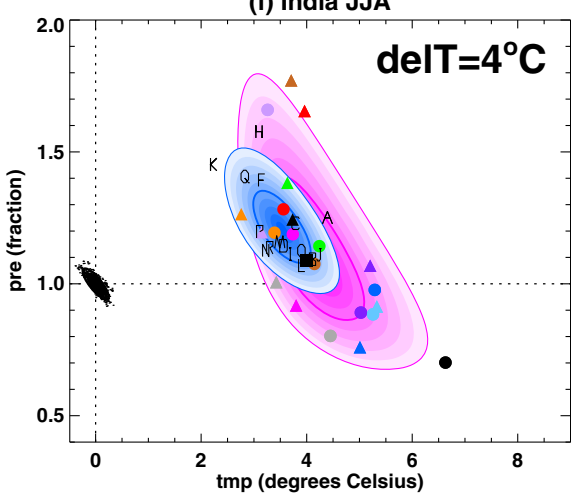

during the summer monsoon season (about $15 \%$ increase for $3{ }^{\circ} \mathrm{C}$ of global warming). A 30year period is often used when considering changes in climate, so it is instructive to compare the pattern-scaled climate changes with inherent climate variability on this timescale. Figure 4 shows the scatter of 30-year mean Indian national-average temperatures and precipitation 
simulated by a 1000-year control run of the UKMO HadCM3 GCM (Tett et al. 2007). Even for $0.5^{\circ} \mathrm{C}$ of global warming, Indian temperature change exceeds the range of 30-year variability, whereas the increase in summer monsoon rainfall does not exceed the variability range until global warming reaches $2{ }^{\circ} \mathrm{C}$. Of course, the range of unforced variability from other GCMs might differ, but UKMO HadCM3 was selected because the availability of a relatively long timeseries reduces the sampling error that is associated with estimating the distribution of 30year means compared with shorter timeseries. It is used indicatively, rather than for any test of statistical significance.

This Indian analysis is extended in Fig. 4c,d to include results from all 22 CMIP3 GCMs available in ClimGen, though only for global warmings of 1 and $4{ }^{\circ} \mathrm{C}$ for clarity. There is considerable spread between GCMs in both the temperature and precipitation changes. In particular, the JJA change (covering most of the summer monsoon period) spans from considerable reductions in mean rainfall (e.g. a $30 \%$ decrease under $4{ }^{\circ} \mathrm{C}$ of global warming) to very large increases (e.g. a $75 \%$ increase under the same warming). The mean and the median of the CMIP3 GCMs both show an increase, but it is worth noting that the Indian average change in summer rainfall can be sensitive to the method used to apply the change patterns (Eqs. 2, 3 or 4): multiplying (as here) the observed reference-period mean by the fractional change in precipitation simulated by the GCM or adding (not shown) the simulated precipitation changes to the observed reference level. The two methods give similar results where a GCM simulates the present-day climate accurately, but can differ where this is not the case.

In Fig. 4e,f we compare these projections for Indian precipitation and temperature from the CMIP3 multi-model ensemble (Table S1) with those derived using patterns diagnosed from a 17-member ensemble of UKMO HadCM3 with perturbed values of some key physical parameters (the QUMP dataset; Murphy et al. 2004). In addition to the individual model results (coloured symbols for CMIP3, black letters and square for QUMP), the shading indicates the \pm 1 and \pm 2 standard deviation (SD) ranges estimated by fitting bivariate normal (or log-normal) distributions to each ensemble of points. These should not be interpreted as probabilistic projections of future change (see Knutti et al. 2010 and Stephenson et al. 2012 for guidance on interpreting model ensembles) but simply as depictions of model spread within the CMIP3 and QUMP ensembles. The QUMP and CMIP3 ensembles yield similar temperature changes in winter (though with a smaller spread in QUMP) but the QUMP summer temperature ensemble lies mostly within the lower half of the CMIP3 ensemble. The ensembles show mostly opposite relative changes in winter precipitation (noting again that these changes are small in absolute terms). In the summer rainfall season, the QUMP ensemble results lie mostly within the greater spread of the CMIP3 ensemble, but all QUMP cases yield an increase in precipitation whereas some CMIP3 models yield summer drying.

The spread of each ensemble, for $4{ }^{\circ} \mathrm{C}$ global warming, far exceeds the HadCM3 estimate of multi-decadal natural variability included in black in this figure. Natural variability would be superimposed on these climate changes for any individual 30-year period. If the inherent variability did not also change with the changing mean climate (Section 2c), and if the UKMO HadCM3 variability is realistic, then each of the symbols representing climate change in Fig. 4 could be replaced by the cloud of 30-year means shown at the origin. It is also interesting to note the similar orientation of natural variability and climate change uncertainty for the summer monsoon season, with higher temperature associated with drier conditions, lower temperatures with wetter conditions. 


\section{Summary}

Like other pattern-scaling approaches, ClimGen attempts to emulate the results of more complex GCMs by separating the geographical, seasonal and multivariate patterns of climate change from their amplitude, with the latter represented by the global-mean temperature change. These techniques have been applied to a range of surface variables required by many climate change impact studies, with normalised patterns of climate change diagnosed from 22 CMIP3 GCMs and 17 perturbed-physics GCMs (QUMP). Recently, we have also diagnosed change patterns from $21 \mathrm{CMIP5}$ GCMs. This approach allows comparison of the multi-model ensemble means and ranges, controlling for scenario and/or global temperature rise, as well as many other applications such as the study of additional mitigation scenarios for which GCM simulations are not available (Arnell et al. 2013). Example results for India (Section 4c) imply that climate impact assessments should not assume that either a multi-model ensemble (e.g. CMIP3) or a perturbed-physics ensemble (e.g. QUMP) is sufficient to represent the full range of future projections. More information about ClimGen is available from http://www.cru.uea. ac.uk/ timo/climgen/.

Pattern scaling can be applied to represent changes in higher-order statistical parameters in addition to the mean climate, and an approach has been reported here to allow GCM-simulated changes in the shape (hence skewness and spread) of the monthly precipitation distribution to be applied to the observed record. This allows the generation of plausible sequences of monthly variability for future climate states, an important development for climate impact studies that depend upon changes in climate variability as well as mean climate. Two approaches for generating multiple realisations can be applied using the ClimGen software, to facilitate the separation of climate change and natural variability effects on climate change impacts. Nevertheless, this approach is not able to represent some changes in the behaviour of natural variability under future climate change, such as a change in the geographical influences of the El Nino-Southern Oscillation (ENSO). To represent such behaviour change (if present) requires the more direct use of GCM simulated climate data, either as simulated or with some elements of model bias compensated by statistical adjustment. However these approaches can only be used for cases with available GCM simulations, whereas GCM emulation approaches such as PS can be used to explore a much wider range of situations and with a wide range of GCMs from multiple ensembles. Ideally, both approaches would be utilised and compared where this is feasible.

Lopez et al. (2014) note some limitations of the PS approach. Their example highlights the need for careful consideration of variability as well as mean climate change, noting reasonable agreement for the frequency of hot summers in 30-year sequences but poor agreement when using 10-year sequences with only one realisation of climate variability. The developments reported here (changing variability of precipitation, which could be adapted for temperature variability, and the ability to generate multiple realisations of variability) address this particular concern. Some limited assessment of the accuracy of the pattern-scaling approximation is reported here, demonstrating that the uncertainty in this approximation is less than the inter-model differences for the CMIP5 ensemble. This uncertainty estimate is conservative, because internal climate variability contaminates the estimated patterns of climate change and thus enhances the difference between patterns diagnosed from separate GCM simulations, and because pattern scaling performance can appear poor where the CMIP5 ensemble spread is narrow. Nevertheless, additional assessment of the performance 
and limitations of the pattern-scaling approach is recommended, adding to recent studies (e.g. Ishizaki et al. 2012; Tebaldi and Arblaster 2014).

Acknowledgments The main development of ClimGen was funded by the UK Natural Environment Research Council (the QUEST-GSI project, NE/E001831/1), building on work funded by the Tyndall Centre for Climate Change Research (IT1.16, T2/11). An extension to include patterns from the CMIP5 model ensemble was supported by the EU TOPDAD project (308620). TJO and TMM were supported by TOPDAD, ICH by QUESTGSI, and CJW by the EU HELIX project (603864). We thank the Met Office Hadley Centre for diagnosing patterns from their QUMP perturbed-physical parameter model ensemble. We acknowledge the modelling groups, the U.S. Department of Energy's Program for Climate Model Diagnosis and Intercomparison (PCMDI), the Global Organization for Earth System Science Portals and the World Climate Research Programme's Working Group on Coupled Modelling for producing and making available the CMIP3 and CMIP5 model output. We thank Tim Mitchell, Nigel Arnell and Rachel Warren for discussion and support over many years and two reviewers for excellent suggestions for improvement.

Open Access This article is distributed under the terms of the Creative Commons Attribution 4.0 International License (http://creativecommons.org/licenses/by/4.0/), which permits unrestricted use, distribution, and reproduction in any medium, provided you give appropriate credit to the original author(s) and the source, provide a link to the Creative Commons license, and indicate if changes were made.

\section{References}

Arnell NW, Lowe JA, Brown S, Gosling SN, Gottschalk P, Hinkel J, Lloyd-Hughes B, Nicholls RJ, Osborn TJ, Osborne TM, Rose GA, Smith P, Warren R (2013) A global assessment of the effects of climate policy on the impacts of climate change. Nat Clim Chang 3:512-519. doi:10.1038/NCLIMATE1793

Arnell N, Brown S, Gosling S, Hinkel J, Huntingford C, Lloyd-Hughes B, Lowe J, Osborn T, Nicholls R, Zelazowski P (2014) Global-scale climate impact functions: the relationship between climate forcing and impact. Clim Chang. doi:10.1007/s10584-013-1034-7

Cabré MF, Solman SA, Nuñez MN (2010) Creating regional climate change scenarios over southern South America for the 2020's and 2050's using the pattern scaling technique: validity and limitations. Clim Chang 98:449-469. doi:10.1007/s10584-009-9737-5

Christensen JH et al (2013) Climate phenomena and their relevance for future regional climate change. In: Climate change 2013: the physical science basis, contribution of Working Group I to the Fifth Assessment Report of the Intergovernmental Panel on Climate Change [Stocker at al. (eds.)]. Cambridge University Press, Cambridge, United Kingdom and New York, NY, USA. doi:10.1017/CBO9781107415324.028

Harris I, Jones PD, Osborn TJ, Lister DH (2014) Updated high-resolution grids of monthly climatic observations - the CRU TS3.10 dataset. Int J Climatol 34:623-642. doi:10.1002/joc.3711

Heinke J, Ostberg S, Schaphoff S, Frieler K, Mueller C, Gerten D, Meinshausen M, Lucht W (2013) A new climate dataset for systematic assessments of climate change impacts as a function of global warming. Geosci Model Dev 6:1689-1703. doi:10.5194/gmd-6-1689-2013

Huntingford C, Booth BBB, Sitch S, Gedney N, Lowe JA, Liddicoat SK, Mercado LM, Best MJ, Weedon GP, Fisher RA, Lomas MR, Good P, Zelazowski P, Everitt AC, Spessa AC, Jones CD (2010) IMOGEN: an intermediate complexity model to evaluate terrestrial impacts of a changing climate. Geosci Model Dev 3: 679-687. doi:10.5194/gmd-3-679-2010

Ishizaki Y, Shiogama H, Emori S, Yokohata T, Nozawa T, Ogura T, Abe M, Yoshimori M, Takahashi K (2012) Temperature scaling pattern dependence on representative concentration pathway emission scenarios. Clim Chang 112:535-546. doi:10.1007/s10584-012-0430-8

Ishizaki Y, Yokohata T, Emori S, Shiogama H, Takahashi K, Hanasaki N, Nozawa T, Ogura T (2014) Validation of a pattern scaling approach for determining the maximum available renewable freshwater resource. $\mathrm{J}$ Hydrometeorol 15:505-516. doi:10.1175/JHM-D-12-0114.1

Knutti R, Abramowitz G, Collins M, Eyring V, Gleckler PJ, Hewitson B, Mearns L (2010) Good practice guidance paper on assessing and combining multi model climate projections. In: Meeting report of the Intergovernmental Panel on Climate Change Expert Meeting on Assessing and Combining Multi Model Climate Projections [Stocker et al. (eds.)]. IPCC Working Group I Technical Support Unit, University of Bern, Bern, Switzerland 
Lopez A, Suckling EB, Smith LA (2014) Robustness of pattern scaled climate change scenarios for adaptation decision support. Clim Chang 122:555-566. doi:10.1007/s10584-013-1022-y

Meehl G, Covey C, Taylor KE, Delworth T, Stouffer RJ, Latif M, McAvaney B, Mitchell JFB (2007) The WCRP CMIP3 multimodel dataset: a new era in climate change research. Bull Am Meteorol Soc 88:1383-1394. doi:10.1175/BAMS-88-9-1383

Mitchell TD (2003) Pattern scaling: an examination of the accuracy of the technique for describing future climates. Clim Chang 60:217-242. doi:10.1023/A:1026035305597

Mitchell JFB, Johns TC, Eagles M, Ingram WJ, Davis RA (1999) Towards the construction of climate change scenarios. Clim Chang 41:547-581. doi:10.1023/A:1005466909820

Murphy JM, Sexton DMH, Barnett DN, Jones GS, Webb MJ, Collins M, Stainforth DA (2004) Quantification of modelling uncertainties in a large ensemble of climate change simulations. Nature 430:768-772. doi:10. 1038/nature02771

Murphy JM, Sexton DMH, Jenkins GJ, Boorman PM, Booth BBB, Brown CC, Clark RT, Collins M, Harris GR, Kendon EJ, Betts RA, Brown SJ, Howard TP, Humphrey KA, McCarthy MP, McDonald RE, Stephens A, Wallace C, Warren R, Wilby R, Wood RA (2009) UK climate projections science report: climate change projections. Met Office Hadley Centre, Exeter

New M, Hulme M (2000) Representing uncertainty in climate change scenarios: a Monte-Carlo approach. Integr Assess 1:203-213. doi:10.1023/A:1019144202120

Osborn TJ (1997) Areal and point precipitation intensity changes: implications for the application of climate models. Geophys Res Lett 24:2829-2832. doi:10.1029/97GL02976

Rayner NA, Parker DE, Horton EB, Folland CK, Alexander LV, Rowell DP, Kent EC, Kaplan A (2003) Global analyses of sea surface temperature, sea ice, and night marine air temperature since the late nineteenth century. J Geophys Res 108:4407. doi:10.1029/2002JD002670

Santer BD, Wigley TML, Schlesinger ME, Mitchell JFB (1990) Developing climate scenarios from equilibrium GCM results. MPI report, 47, Max Planck Institute for Meteorology, Hamburg, Germany

Stephenson DB, Collins M, Rougier JC, Chandler RE (2012) Statistical problems in the probabilistic prediction of climate change. Environmetrics 23:364-372. doi:10.1002/env.2153

Taylor KE, Stouffer RJ, Meehl GA (2012) An overview of CMIP5 and the experimental design. Bull Am Meteorol Soc 93:485-493. doi:10.1175/BAMS-D-11-00094.1

Tebaldi C, Arblaster JM (2014) Pattern scaling: its strengths and limitations, and an update on the latest model simulations. Clim Chang 122:459-471. doi:10.1007/s10584-013-1032-9

Tett SFB, Betts R, Crowley TJ, Gregory J, Johns TC, Jones A, Osborn TJ, Ostrom E, Roberts DL, Woodage MJ (2007) The impact of natural and anthropogenic forcings on climate and hydrology since 1550. Clim Dyn 28:3-34. doi:10.1007/s00382-006-0165-1

van Vuuren DP et al (2011) The representative concentration pathways: an overview. Clim Chang 109:5-31. doi: $10.1007 / \mathrm{s} 10584-011-0148-\mathrm{Z}$

Warren R, de la Nava SS, Arnell NW, Bane M, Barker T, Barton C, Ford R, Fuessel H-M, Hankin RKS, Klein R, Linstead C, Kohler J, Mitchell TD, Osborn TJ, Pan H, Raper SCB, Riley G, Schellnhuber HJ, Winne S, Anderson D (2008) Development and illustrative outputs of the Community Integrated Assessment System (CIAS), a multi-institutional modular integrated assessment approach for modelling climate change. Environ Model Softw 23:592-610. doi:10.1016/j.envsoft.2007.09.002

Warren R, Lowe JA, Arnell NW, Hope C, Berry P, Brown S, Gambhir A, Gosling SN, Nicholls RJ, O’Hanley J, Osborn TJ, Osborne T, Price J, Rose G, Vanderwal J (2013) The AVOID programme's new simulations of the global benefits of stringent climate change mitigation. Clim Chang 120:55-70. doi:10.1007/s10584-0130814-4

Watterson I, Whetton P (2011) Joint PDFs for Australian climate in future decades and an idealized application to wheat crop yield. Aust Meteorol Oceanogr J 61:221-230 